\title{
High-flux X-ray photon emission by a superluminal hybrid electromagnetic mode of intense laser in a plasma waveguide
}

\author{
J. Wang ${ }^{1}{ }^{2}$ (王剑), B. $\mathrm{Zhu}^{3}$ (朱斌), T. P. Yu (余同普), Z. Q. \\ Zhao $^{3}$ (赵宗清), Z. M. Sheng ${ }^{2}$ (盛政明), Y. Q. Gu ${ }^{3}$ (谷渝秋) \\ ${ }^{1}$ College of Physics and Electronic Information Engineering, Neijiang Normal \\ University, Neijiang, 641000, People's Republic of China \\ 2 SUPA Department of Physics, Strathclyde University, Glasgow, G4 0NG, UK \\ ${ }^{3}$ National Key Laboratory of High-Temperature and High-Density Plasma Physics, \\ Mianyang, 621900, People's Republic of China \\ ${ }^{4}$ College of Arts and Sciences, National Defense Science and Technology University, \\ Changsha , 410073, People's Republic of China \\ E-mail: yqgu@caep.ac.cn
}

May 2019

\begin{abstract}
The feasibility of several novel ultrafast X-ray sources has been demonstrated through the interaction between laser and a micro-structured target. However, the resulting photon flux is still deficient for applications. Here, we proposed a new method to yield high-flux synchrotron radiation by adopting a compact hollow plasma waveguide. Linearly polarized laser pulses propagating in the waveguide are mainly converted into the electromagnetic mode $\mathrm{HE}_{11}$ with a superluminal phase speed of $1.02 c$ when the pulse is coupled with the waveguide. The mode fields lead to a strong oscillating force and a short oscillation period for energetic electrons, which are accelerated via the longitudinal acceleration mechanism. Then, short X-ray pulses are generated with high yield of $10^{11}-10^{12}$. The corresponding photon flux reaches $3.5 \times 10^{21}$ photons $/ \mathrm{s} \cdot 0.1 \%$ bandwidth. High-flux source can be used for single shot ultrafast imaging or the ultrafast X-ray diffraction and absorption studies.
\end{abstract}

PACS numbers: 42.81.Qb, 41.75.Jv, 41.60.Ap

Keywords: Higher-order mode Synchrotron radiation High-flux

Submitted to: Plasma Phys. Control. Fusion 


\section{Introduction}

High-energy radiation can be delivered by radioactive sources, X-ray tubes, and Compton scattering sources based on conventional accelerators. Even though these sources are widely used, they have limitations in terms of storage, pulse duration, spectrum tunability, energy range, and source size. Hence, novel radiation sources are being developed, e.g., betatron synchrotron radiation sources are generated in the laser-driven wakefield electron acceleration (LWFA). Many theoretical and experimental results have demonstrated the feasibility of these sources based on the LWFA [1, $2,3,4,5,6,7,8,9,10]$. However, their application in probing dense matter is limited owing to a low energy efficiency and photon energy in the range of tens of kiloelectronvolt. Meanwhile, it has been reported that a laser-ion channel (LIC) is also used to produce a plasma wiggler with a considerably higher strength parameter than the LWFA regime. This leads to an X-ray source that extends to much higher energies than those achieved previously, with the benefits of brightness, short pulse duration, and laser synchronization with other all-optical synchrotron sources [11, 12, 13, 14]. Even a radiation source with flexible tunabilities can be realized based on an LIC [15, 16]. Usually, these radiation sources have large divergence in the LIC $[12,13]$.

The advancements in the laser and material science fields have made it possible to overcome the above mentioned limitations. Recently, the feasibility of manipulating high intensity laser beam has been demonstrated through a micro-structured target $[17,18,19,20,21]$. Further, some synchrotron radiation sources based on the microstructured target also have been proposed directly [22, 23]. The emission of high-energy $(>10 \mathrm{keV})$ photons attains a maximum conversion efficiency of approximately $10 \%$ at $1 \times 10^{22} \mathrm{~W} / \mathrm{cm}^{2}$ because a PW laser pulse is coupled into the nanowire target [22]. Moreover, the photon yield is found to be comparable with that achieved in a uniform plasma of same average density; however, nanowire arrays provide efficient radiation sources over a broader parameter range.

In this paper, we report a novel method to produce a brilliant synchrotron radiation source from a compact hollow waveguide. Approximately $10^{11}-10^{12}$ photons are obtained in the waveguide of length $20 \mu \mathrm{m}$. Through three-dimensional particle-incell simulations, it is found that a linearly polarized laser pulse is mainly converted to the electromagnetic mode $\mathrm{HE}_{11}$ with high phase speed inside the waveguide. The electrsomagnetic mode traps and accelerates electrons to superponderomotive energies through the longitudinal field components. The accelerated electrons are overtaken by the transverse fields with the superluminal phase speed and wiggling with strong transverse forces and short oscillation periods. As a result, an X-ray beam with high flux and moderate divergence is produced. The typical photon flux of the synchrotron radiation is $3.5 \times 10^{21}$ photons $/ \mathrm{s} \cdot 0.1 \%$ bandwidth, and the corresponding spectral brightness is $3.7 \times 10^{22}$ photons $/ \mathrm{s} \cdot \mathrm{mrad}^{2} \cdot \mathrm{mm}^{2} \cdot 0.1 \%$ bandwidth with $100 \%$ energy spread.

The remainder of this paper is organized as follows. In Sec.2, the mode conversion of 


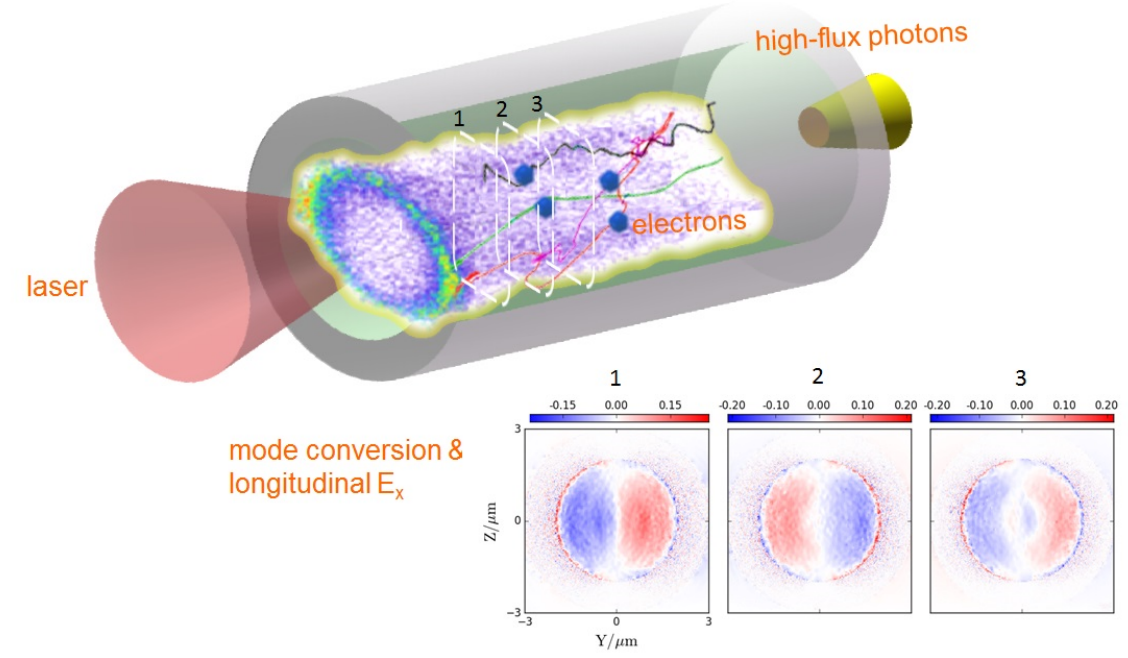

Figure 1. Schematic of high-flux synchrotron radiation from a compact hollow waveguide. Insets 1-3 represent the mode conversion of the laser fields. Therefore, a considerable number of electrons are sufficiently accelerated by the longitudinal laser fields. Then, they wiggle in the transverse fields and experience intense transverse oscillations at the high phase speed. These oscillations of the energetic electrons tend to generate high X-ray photon flux.

a linearly polarized pulse in the compact hollow waveguide is proposed and investigated through three-dimensional particle-in-cell simulations. In Sec.3 and Sec.4, the radiation properties and their tunabilities are discussed, respectively. Finally, Sec.5 concludes the paper.

\section{Propagation of a high intensity laser pulse in a compact hollow waveguide}

Figure.1 shows a schematic of high-flux synchrotron radiation from a compact hollow waveguide. The compact hollow waveguide is likely to be produced by guiding a lowlevel prepulse through a tube inside a photonic crystal fiber[24, 25]. A short pulse with the intensity of $3 \times 10^{20} \mathrm{~W} / \mathrm{cm}^{2}$ is coupled into the waveguide, and the diameter is comparable to the laser waist size. It is well known that the laser pulse cannot propagate in the transverse electromagnetic mode inside the waveguide. Instead, as the subplots 1,2,3 in Fig.1 show, mode conversion into other electromagnetic modes is inevitable, including the HE mode, EH mode, TE mode, TM mode, etc[26]. The conversion is highly complicated and difficult to predict directly. Thus, to understand the underlying phenomena, we performed three-dimensional particle-in-cell simulations with the open source code EPOCH[27].

The simulation box size is $24 \lambda \times 10 \lambda \times 10 \lambda$ with a mesh grid of $600 \times 300 \times 300$ in the $\mathrm{x}, \mathrm{y}$, and $\mathrm{z}$ directions, where $\lambda=1 \mu \mathrm{m}$ is the laser wavelength. A full-ionized 
High-flux X-ray photon emission by a superluminal hybrid electromagnetic mode of intense laser in a plasma
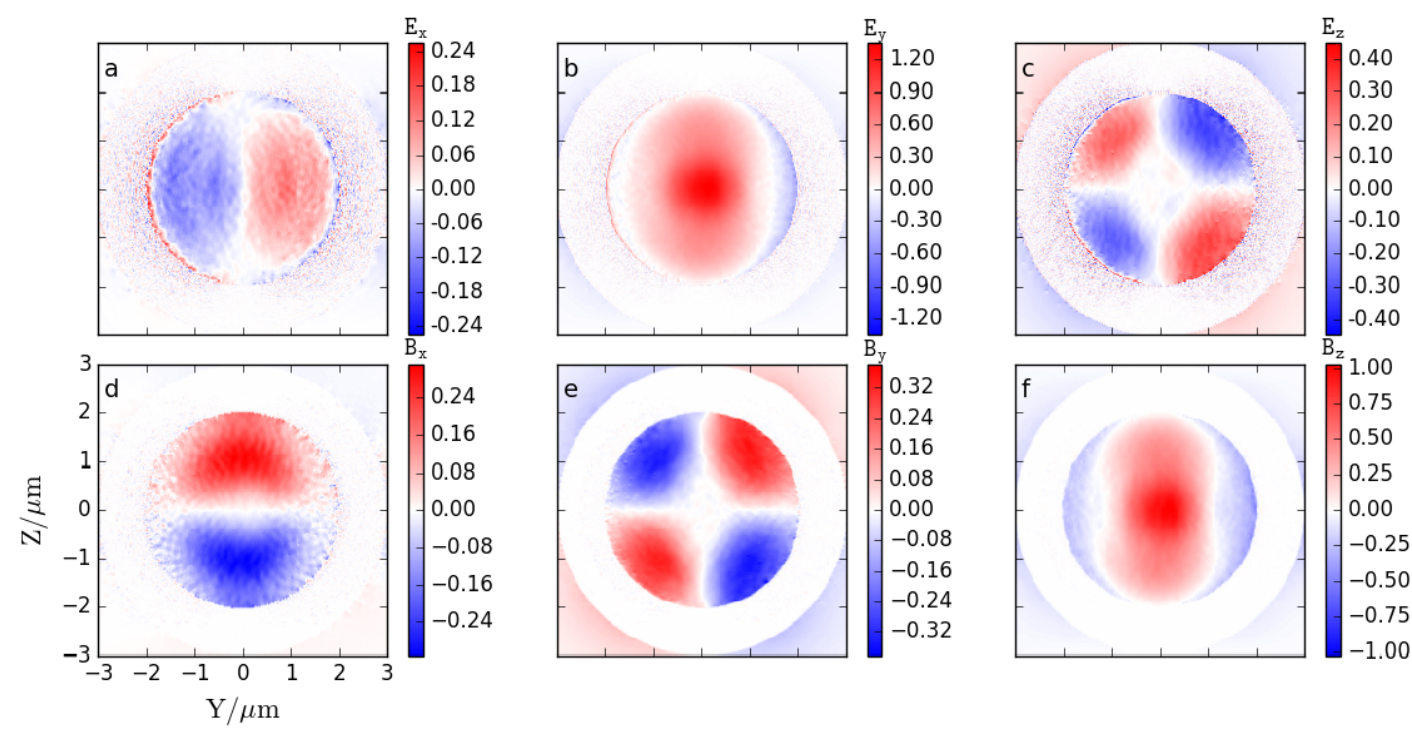

Figure 2. Laser field components in the cross-section $x=7 \mu \mathrm{m}$ at $t=15 T$. The upper row shows the $E_{x}(\mathrm{a}), E_{y}(\mathrm{~b})$ and $E_{z}(\mathrm{c})$, and the lower row shows the $B_{x}(\mathrm{~d}), B_{y}(\mathrm{e})$ and $B_{z}(\mathrm{f})$. The electric components are normalized by $m_{0} \omega_{0} c / e$, and the magnetic components are normalized by $m_{0} \omega_{0} / e$, where $\omega_{0}$ is the angular frequency of the laser, $m_{0}$ is electron mass, $e$ is electron charge, and $c$ is the light speed.

waveguide is placed in the middle of the simulation box. There is a vacuum gap of $5 \mu \mathrm{m}$ between the waveguide and the left boundary of the simulation domain. The ion density $n_{i}$ of $\mathrm{C}^{6+}$ is $16.6 n_{c}$, corresponding to an electron density $n_{e}$ of $100 n_{c}$, where $n_{c}$ is the critical density. The radius of the waveguide and the thickness of the wall are $r_{0}=2 \mu \mathrm{m}$ and $1 \mu \mathrm{m}$, respectively. The initial ion and electron temperature are both set as $10 \mathrm{eV}$. A $p$-polarized Gaussian laser with the vector potential $a=a_{0} \exp \left[-\left(y^{2}+z^{2}\right) / w_{0}^{2}-\left(t-2 \tau_{0}\right)^{2} / \tau_{0}^{2}\right]$ is incident to the left boundary of the simulation domain at $0^{\circ}$, where $a_{0}=15, w_{0}=3 \lambda, \tau_{0}=9 T$, and $T$ is the laser period.

As shown in Fig. 2, there are six normalized field components in the cross-section $x=7 \mu \mathrm{m}$ at $t=15 \mathrm{~T}$. Considering the strong skin effect caused by the overdense wall shown in Fig.3, the hollow waveguide is similar to a metallic waveguide with dielectric constant $\epsilon \rightarrow-\infty$ at the earlier stage of the simulation. Thus, these components in Fig. 2 are equivalent to a linear combination of the TE(H) and TM(E) modes[26]. For simplicity, we assume that the incident wave is planar in our further discussion. The corresponding TE and TM fields inside the waveguide can be expressed as[26]

$$
\begin{aligned}
& c B_{x}^{h}=\sum_{\mu, \nu} a_{\mu \nu} J_{\mu}\left(\kappa_{\mu \nu} r\right) \sin (\mu \phi) e^{i k_{x} x}+c . c ., \\
& c B_{r}^{h}=\sum_{\mu, \nu}-i a_{\mu \nu} \frac{k_{x}}{\kappa_{\mu \nu}^{2} r} \mu J_{\mu}\left(\kappa_{\mu \nu} r\right) \sin (\mu \phi) e^{i k_{x} x}+c . c ., \\
& c B_{\phi}^{h}=\sum_{\mu, \nu}-i a_{\mu \nu} \frac{k_{x}}{\kappa_{\mu \nu}} J_{\mu}^{\prime}\left(\kappa_{\mu \nu} r\right) \cos (\mu \phi) e^{i k_{x} x}+c . c .,
\end{aligned}
$$


High-flux X-ray photon emission by a superluminal hybrid electromagnetic mode of intense laser in a plasma
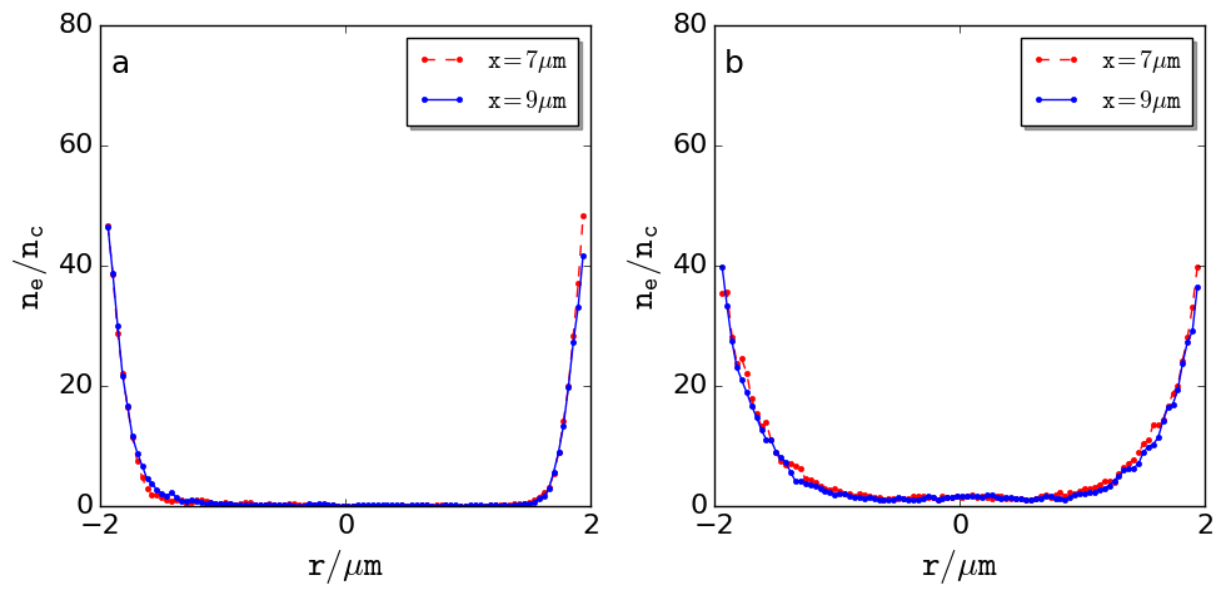

Figure 3. Radial averaged electron density distributions in the different cross-sections at $t=15 T(\mathrm{a})$ and $t=30 T(\mathrm{~b})$.

$$
\begin{aligned}
& E_{r}^{h}=\sum_{\mu, \nu}-i a_{\mu \nu} \frac{k}{\kappa_{\mu \nu}^{2} r} \mu J_{\mu}\left(\kappa_{\mu \nu} r\right) \cos (\mu \phi) e^{i k_{x} x}+c . c . \\
& E_{\phi}^{h}=\sum_{\mu, \nu} i a_{\mu \nu} \frac{k}{\kappa_{\mu \nu}} J_{\mu}^{\prime}\left(\kappa_{\mu \nu} r\right) \sin (\mu \phi) e^{i k_{x} x}+c . c .
\end{aligned}
$$

and

$$
\begin{aligned}
& E_{x}^{e}=\sum_{\mu, \nu} b_{\mu \nu} J_{\mu}\left(\kappa_{\mu \nu}^{\prime} r\right) \cos (\mu \phi) e^{i k_{x}^{\prime} x}+c . c . \\
& E_{r}^{e}=\sum_{\mu, \nu}-i b_{\mu \nu} \frac{k_{x}}{\kappa_{\mu \nu}^{\prime}} J_{\mu}^{\prime}\left(\kappa_{\mu \nu}^{\prime} r\right) \cos (\mu \phi) e^{i k_{x}^{\prime} x}+c . c ., \\
& E_{\phi}^{e}=\sum_{\mu, \nu} i b_{\mu \nu} \frac{k_{x}}{\kappa_{\mu \nu}^{\prime}{ }^{2} r} \mu J_{\mu}\left(\kappa_{\mu \nu}^{\prime} r\right) \sin (\mu \phi) e^{i k_{x}^{\prime} x}+c . c ., \\
& c B_{r}^{e}=\sum_{\mu, \nu}-i b_{\mu \nu} \frac{k}{\kappa_{\mu \nu}^{\prime}{ }^{2} r} \mu J_{\mu}\left(\kappa_{\mu \nu}^{\prime} r\right) \sin (\mu \phi) e^{i k_{x}^{\prime} x}+c . c . \\
& c B_{\phi}^{e}=\sum_{\mu, \nu}-i b_{\mu \nu} \frac{k}{\kappa_{\mu \nu}^{\prime}} J_{\mu}^{\prime}\left(\kappa_{\mu \nu}^{\prime} r\right) \cos (\mu \phi) e^{i k_{x}^{\prime} x}+c . c .
\end{aligned}
$$

where $r=\sqrt{y^{2}+z^{2}}, \kappa_{\mu \nu}\left(\kappa_{\mu \nu}^{\prime}\right)=\sqrt{k^{2}-k_{x}^{2}\left(k_{x}^{\prime 2}\right)}, \phi=[0-2 \pi], a_{\mu \nu}, b_{\mu \nu}$ are the coefficients, $\mu, \nu$ are the subindices of the modes, $k, k_{x}$ are the wave number and wave number in $x$ direction, respectively, and $J_{\mu}$ is the Bessel function of the first kind. From the transverse electric field or the magnetic field components illustrated in Fig.2, the modes in which the laser pulse operates can be deduced. It is clear that the patterns of the $E_{y}=\left(E_{r}^{e}+E_{r}^{h}\right) \cos \phi-\left(E_{\phi}^{e}+E_{\phi}^{h}\right) \sin \phi$ and $E_{z}=\left(E_{r}^{e}+E_{r}^{h}\right) \sin \phi+\left(E_{r}^{e}+E_{r}^{h}\right) \cos \phi$ in Fig. 2 can be a linear combination of $\mathrm{TE}_{11}, \mathrm{TE}_{12}$ and $\mathrm{TM}_{11}$ mode. These modes are separated from each other for the different longitudinal wavenumbers. 
High-flux X-ray photon emission by a superluminal hybrid electromagnetic mode of intense laser in a plasma

However, as shown in Fig. 3, considerable number of electrons are pulled out and piled on the wall of the waveguide by the laser fields, resulting in a dielectric structure with graded refraction index because of the leading part of the laser pulse. Thus, the waveguide behaves like a dielectric waveguide. One can predict that the hybrid electromagnetic mode should dominate in the later stage[26], instead of the $\mathrm{TM}_{11}$ mode $[28,29]$. The corresponding fields inside the plasma waveguide can be changed to the following

$$
\begin{aligned}
E_{x} & =\sum_{m, n} a_{m n} \kappa_{m n}^{2} J_{m}\left(\kappa_{m n} r\right) \cos (m \phi) e^{i k_{x} x}+c . c ., \\
B_{x} & =\sum_{m, n} \mu_{0} b_{m n} \kappa_{m n}^{2} J_{m}\left(\kappa_{m n} r\right) \sin (m \phi) e^{i k_{x} x}+c . c ., \\
E_{r} & =-i \sum_{m, n}\left[a_{m n} k_{x} \kappa_{m n} J_{m}^{\prime}\left(\kappa_{m n} r\right)\right. \\
& \left.+b_{m n} \mu_{0} \omega_{0} m \frac{J_{m}\left(\kappa_{m n} r\right)}{r}\right] \cos (m \phi) e^{i k_{x} x}+c . c . \\
E_{\phi} & =i \sum_{m, n}\left[b_{m n} \mu_{0} \omega_{0} \kappa_{m n} J_{m}^{\prime}\left(\kappa_{m n} r\right)\right. \\
& \left.+a_{m n} k_{x} m \frac{J_{m}\left(\kappa_{m n} r\right)}{r}\right] \sin (m \phi) e^{i k_{x} x}+c . c ., \\
B_{r} & =-i \mu_{0} \sum_{m, n}\left[b_{m n} k_{x} \kappa_{m n} J_{m}^{\prime}\left(\kappa_{m n} r\right)\right. \\
& \left.+a_{m n} \omega_{0} \epsilon_{0} m \frac{J_{m}\left(\kappa_{m n} r\right)}{r}\right] \sin (m \phi) e^{i k_{x} x}+c . c ., \\
B_{\phi} & =-i \mu_{0} \sum_{m, n}\left[a_{m n} \omega_{0} \epsilon_{0} \kappa_{m n} J_{m}^{\prime}\left(\kappa_{m n} r\right)\right. \\
& \left.+b_{m n} m k_{x} \frac{J_{m}\left(\kappa_{m n} r\right)}{r}\right] \cos (m \phi) e^{i k_{x} x}+c . c .
\end{aligned}
$$

where $\kappa_{m n}=\sqrt{k^{2}-k_{x}^{2}}, a_{m n}, b_{m n}$ are coefficients, $m, n$ are the subindices of the modes, $k, k_{x}$ are the wave number and wave number in $x$ direction, respectively, $J_{m}$ is the Bessel function of the first kind, $\epsilon_{0}, \mu_{0}$ are the vacuum dielectric constant and magnetic permeability, respectively. By substituting $b_{m n}=-a_{m n} k_{x} s / \omega_{0} \mu_{0}$ in Eq. 3, transverse electric field components can be simplified to

$$
\begin{aligned}
c_{m n} & =a_{m n} k_{x} \kappa_{m n}, \\
E_{r} & =-i \sum_{m, n} c_{m n}\left[\frac{1-s}{2} J_{m-1}\left(\kappa_{m n} r\right)\right. \\
& \left.-\frac{1+s}{2} J_{m+1}\left(\kappa_{m n} r\right)\right] \cos (m \phi) e^{i k_{x} x}+c . c ., \\
E_{\phi} & =i \sum_{m, n} c_{m n}\left[\frac{1-s}{2} J_{m-1}\left(\kappa_{m n} r\right)\right.
\end{aligned}
$$


High-flux X-ray photon emission by a superluminal hybrid electromagnetic mode of intense laser in a plasma
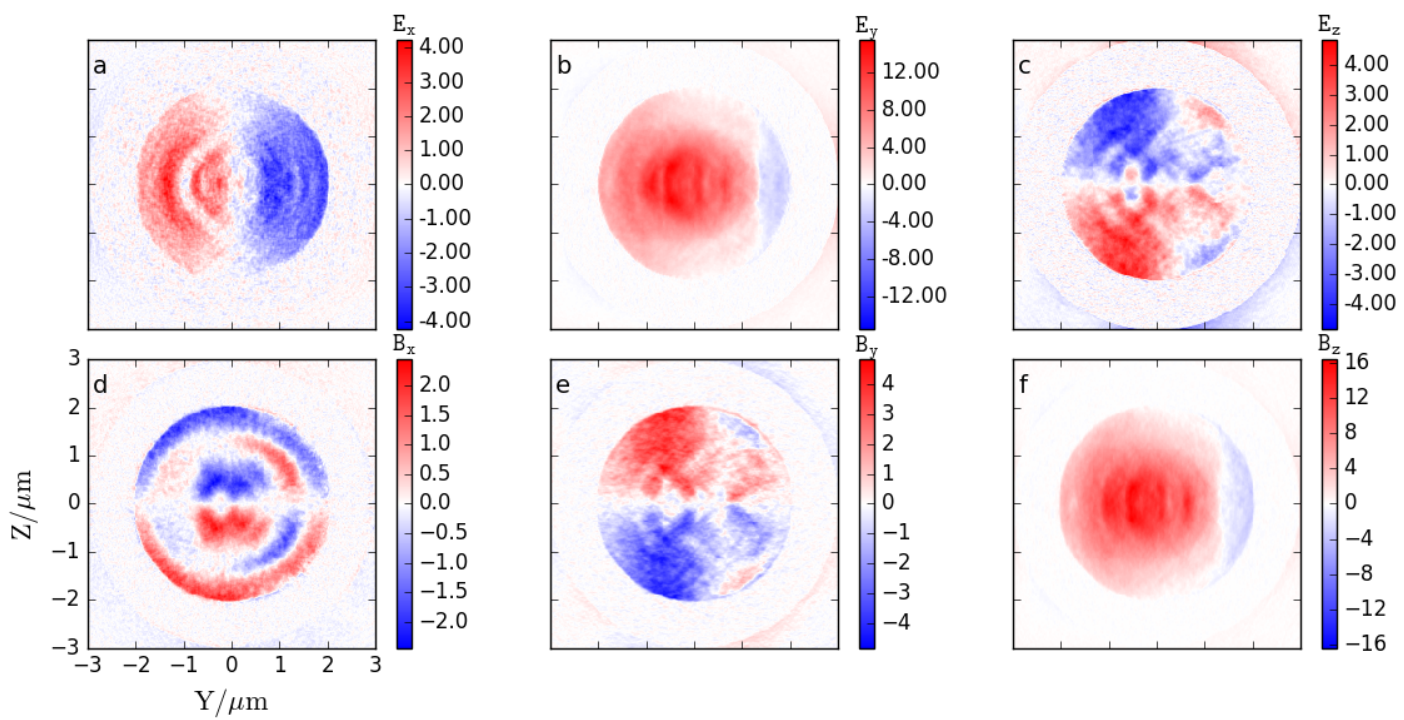

Figure 4. Laser field components in the cross-section $x=7 \mu \mathrm{m}$ at $t=30 T$. The upper row shows $E_{x}(\mathrm{a}), E_{y}(\mathrm{~b})$ and $E_{z}(\mathrm{c})$, and the lower row shows $B_{x}(\mathrm{~d}), B_{y}(\mathrm{e})$ and $B_{z}(\mathrm{f})$. They are normalized by $m_{0} \omega_{0} c / e$, where $\omega_{0}$ is the angular frequency of the laser, $m_{0}$ is electron mass, $e$ is electron charge, and $c$ is the light speed.

$$
\left.-\frac{1+s}{2} J_{m+1}\left(\kappa_{m n} r\right)\right] \sin (m \phi) e^{i k_{x} x}+c . c .
$$

where $s$ is a coefficient. It is clear that the patterns of $E_{y}$ and $E_{z}$ in Fig.4(a), (b), (f) agree well with the $\mathrm{HE}_{11}$ mode in Eq.4 as $s \simeq-1$, i.e., $E_{y}=-i c_{m n}\left[\frac{1-s}{2} J_{0}\left(\kappa_{11} r\right)-\right.$ $\left.\frac{1+s}{2} J_{2}\left(\kappa_{11} r\right) \cos (2 \phi)\right] e^{i k_{x} x}+$ c.c. and $E_{z}=i c_{m n} \frac{1+s}{2} J_{2}\left(\kappa_{11} r\right) \sin (2 \phi) e^{i k_{x} x}+$ c.c.. According to the boundary conditions $J_{0}\left(\kappa_{11} r_{0}\right) \approx 0$, the transverse number $\kappa_{11}=1.2 \times 10^{6} / \mathrm{m}$ and the phase speed $v_{p}=c / \sqrt{1-\left(\kappa_{11} / k\right)^{2}}=1.02 c$ are obtained immediately.

\section{Electron acceleration and synchrotron radiation}

Owing to the simultaneous appearance of the transverse and axial electric fields in Fig.4(a) and (b), some electrons are extracted from the wall of the waveguide by the transverse components and injected into the longitudinal components for the phase difference of $\pi / 2$ between them from Eq. 3. Thus, the energy gain $\Gamma$ of the trapped electrons in the laser field is governed by

$$
\Gamma=\Gamma_{\|}+\Gamma_{\perp}=-e \int_{0}^{t}\left(v_{x} E_{x}+v_{y} E_{y}+v_{z} E_{z}\right) d t,
$$

where the first term is relative to the longitudinal fields $E_{x} \simeq \kappa_{11} a_{0} / k=2.8$ while the last two terms are determined by the transverse fields $E_{y}$ and $E_{z}$. Figure 5(a) shows the electron energy gain from longitudinal components versus that from transverse components at $t=30 T$. The first term of Eq. 5 is the main contributor to the energy 
High-flux X-ray photon emission by a superluminal hybrid electromagnetic mode of intense laser in a plasma
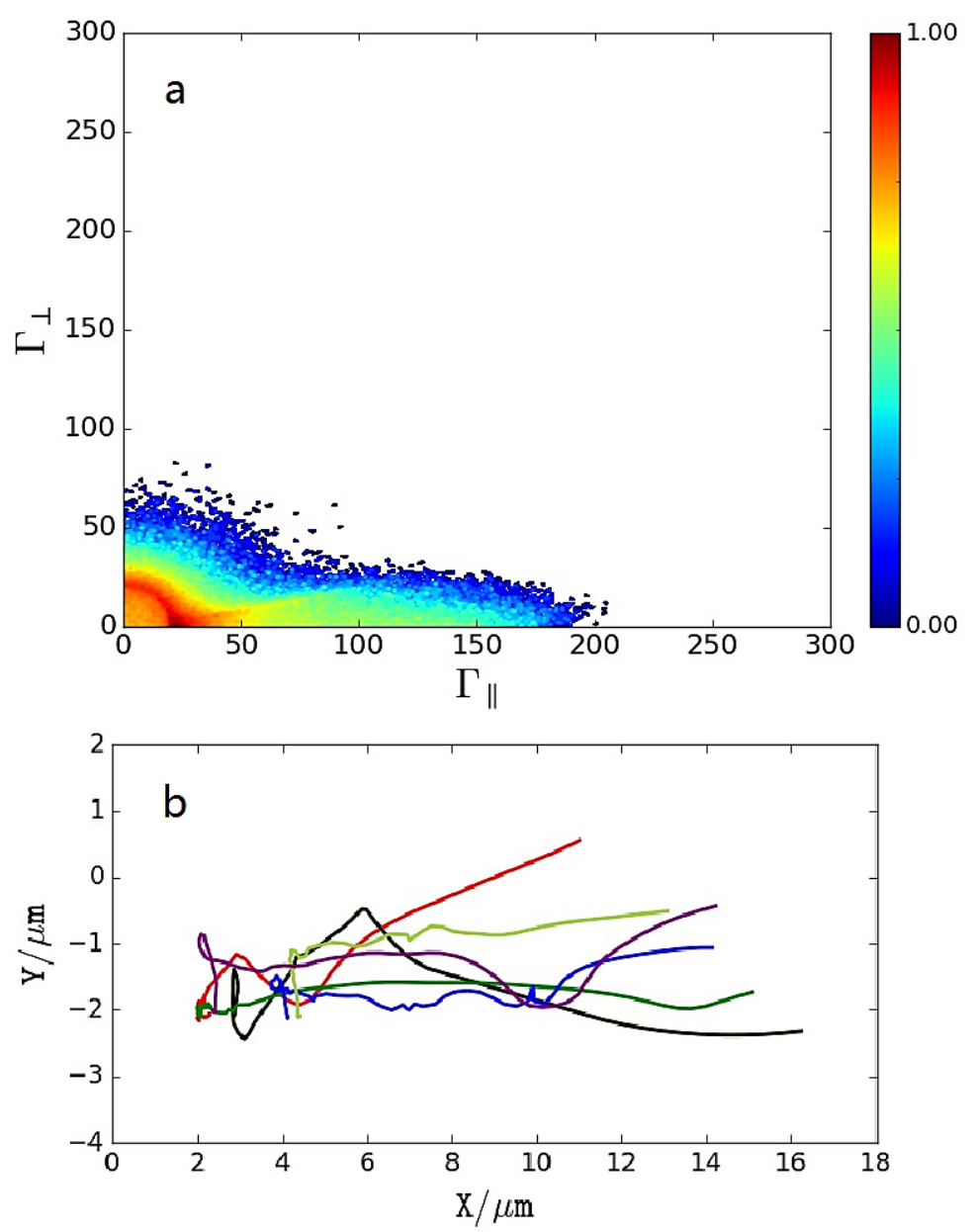

Figure 5. Electron energy gain from longitudinal fields versus that from transverse fields at $t=30 T$; (b) Typical trajectories of energetic electrons.

gain of highly energetic electrons, and there is a small contribution from the transverse field components. The corresponding dephasing length is $1 /\left(1-c / v_{p}\right) \lambda_{0} / 2=25 \mu \mathrm{m}$.

By integrating the energy spectra in Fig.6(a) at the end of the simulation, as many as $7 \times 10^{10}$ energetic electrons $(>10 \mathrm{MeV})$ are achieved. The cutoff of the electron energy spectra in Fig.6(a) exceeds the so-called free electron limit of $\left(1+a_{0}^{2} / 2\right) m_{0} c^{2}[30]$, exceeding $100 \mathrm{MeV}$ at the end of the simulation. Figure.6(b) shows the radial Lorentz force which is seen by the energetic electrons. The maximum of the force is $0.2 m_{0} \omega_{0} c a_{0}$. In fact, the amplitude of the Lorentz force that is perpendicular to the forward moving electrons is

$$
F_{\perp}=e \sqrt{\left(E_{r}-\frac{v_{x}}{c} B_{\phi}\right)^{2}+\left(E_{\phi}+\frac{v_{x}}{c} B_{r}\right)^{2}} .
$$

Substituting Eq.3, $a_{m n}=m_{0} \omega_{0} c a_{0} / e k \kappa_{m n}, b_{m n}=-a_{m n} k_{x} s / \omega_{0} \mu_{0}$ and $s \simeq-1$ in Eq.6, one can see that $F_{\perp} \simeq\left(v_{p} / c-1\right) m_{0} c \omega_{0} a_{0}=0.02 m_{0} c \omega_{0} a_{0}$ qualitatively agrees with the simulation results in Fig.6(b). Both the simulations and the analysis suggest that the accelerated electrons can be overtaken by the mode fields and emit a large number of 
photons.

Figure 6(c) shows the radiation photon spectra in our simulations. Some important parameters, such as strength parameter $K$ can be determined approximately by the synchrotron-like spectra. For the energetic electrons, the typical oscillating period is $\lambda_{u}=1 /\left(1-v_{x} / v_{p}\right) \lambda_{0} / 2 \simeq 1 /\left(1-c / v_{p}\right) \lambda_{0} / 2=25 \mu \mathrm{m}$ and the amplitude of oscillation is $r_{\beta} \sim 1 \mu \mathrm{m}$ from Fig.5(b). As discussed above, $\psi=2 \pi r_{\beta} / \lambda_{u} \approx 0.3, K=\gamma \psi>1$. Thus, the synchrotron radiation should be treated as a wiggler. Based on the wellknown synchrotron function, $d I / d \omega \propto \omega / \omega_{c} \int_{\omega / \omega_{c}}^{\infty} K_{5 / 3}\left(\omega / \omega_{c}\right) d \omega / \omega_{c}[7]$, where $K_{5 / 3}$ is the modified Bessel function of second kind, and $\omega_{c}$ is the critical frequency of the spectra. However, it is difficult to identify the critical energies $\hbar \omega_{c}$ in the spectra because of their deviation from the classic synchrotron radiation spectrum. Then, the average photon energy $\langle\hbar \omega\rangle$ is employed to estimate the photon yield. The number of photons emitted per period and per electron at the average photon energy $\langle\hbar \omega\rangle$ scales is $N=0.033 K[7]$. The theoretical yield of the $\mathrm{X}$-ray photons should be on the order of $10^{11}$ for one oscillating period. This agrees with the value of $1.7 \times 10^{11}$ from the simulations. In contrast, for a $100-\mathrm{MeV}$ wakefield electron beam undergoing betatron oscillations in a plasma with a density of $2 \times 10^{19} \mathrm{~cm}^{-3}$, the oscillation period is $\lambda_{\mathrm{u}}=150$ $\mu \mathrm{m}$. The critical energy of the radiation $\hbar \omega_{\mathrm{c}}$ can reach $5 \mathrm{keV}$ when $\mathrm{K} \sim 10$. The number of expected X-ray photons is in the range of $10^{8-9}[7,2]$. It is clear that the number of X-ray photons from the waveguide is more than two orders of magnitude higher than the photons from laser-wakefield acceleration. In Fig.6(d), we present the energy radiated per unit solid angle $d I / d \Omega$. One can see that most of the emissions are within $5^{\circ}$, which is much smaller than the typical results, 0.87-1.57 radian, in the laser-ion channel[12, 13]. This implies that the photon flux of the synchrotron radiation is equal

to $3.5 \times 10^{21}$ photons $/ \mathrm{s} \cdot 0.1 \%$ bandwidth, and the spectral brightness is $3.7 \times 10^{22}$ photons $/ \mathrm{s} \cdot \mathrm{mrad}^{2} \cdot \mathrm{mm}^{2} \cdot 0.1 \%$ bandwidth with $100 \%$ energy spread. This value is comparable to the current third-generation conventional wiggler light sources[7, 8].

\section{Tunabilities of the radiation}

From Fig.7(a), the fitting shows that the total yields are dependent on $a_{0}^{3}$. The number of energetic electrons is mainly determined by the laser energy. However, in contrast with our expectations, the average photon energies $\langle\hbar \omega\rangle$ scales as $\omega_{c} \propto a_{0}^{2.4}$, deviate from the $\omega_{c} \propto a_{0}^{3}$ scaling law. Because of the nonlinear evolution in Fig.8(a)-(c), the complicated behavior of the mode fields may lead the scaling law to deviate from the cubic form. Finally, we explore the dependence of the yields on the diameter of the waveguide. In Fig.7(b), the yields start decreasing gradually, however, the average photon energies continue to increase with the diameter for $d>4 \mu \mathrm{m}$. In particular, it is interesting that the yields fall to the order of $10^{10}$ despite the average photon energy increasing to 28 $\mathrm{keV}$. It can be concluded that the optimal diameter of the waveguide is comparable to the size of the laser waist $w_{0}$ for high-flux output. When the compact hollow waveguide is adopted, the intense laser-wall interactions tend to construct a dielectric structure 

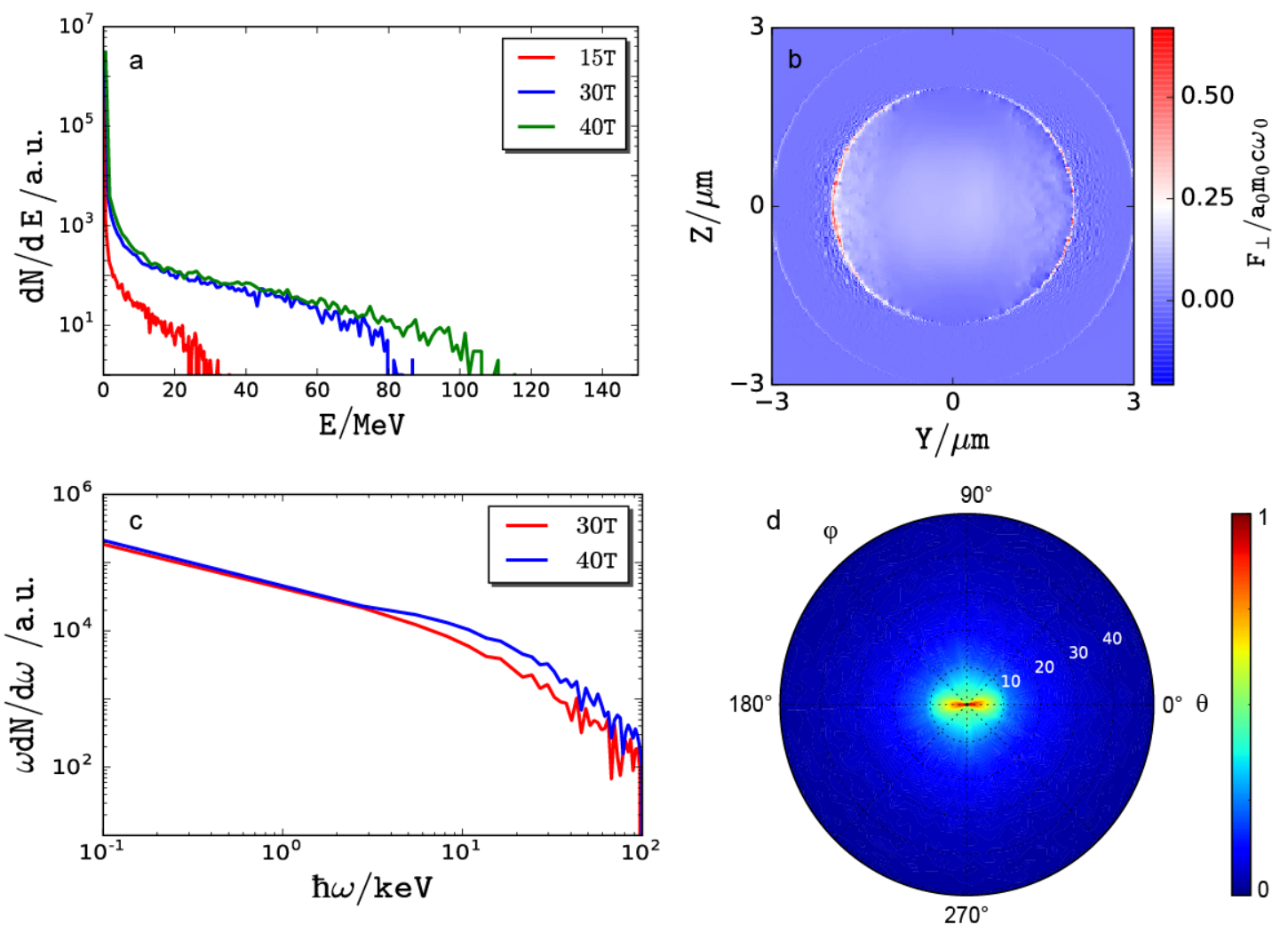

Figure 6. (a) Electron energy spectra at different times; (b) Transverse net force $F_{\perp}$ in the cross-section $x=7 \mu \mathrm{m}$ at $t=30 T$, which is normalized by $m c \omega_{0} a_{0}$; (c) Photon energy spectra at different times, the green dashed line represents the power fitting; (d) Angular distribution of the synchrotron radiation photons at the end of the simulation. $\theta$ is the angle between the photon emission direction and the $x$ axis, $\phi$ is the angle between the photon emission direction and the $y$ axis in the $y-z$ plane.
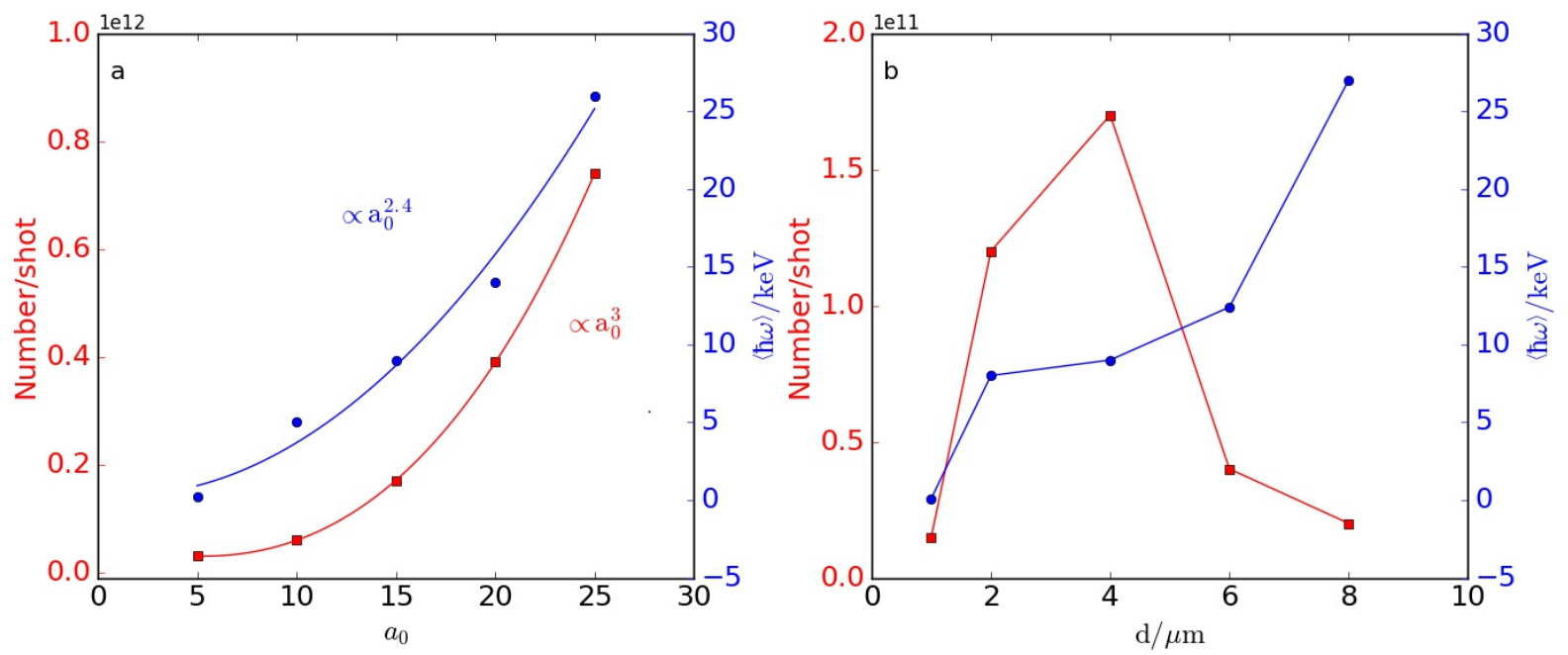

Figure 7. Dependence of the photon yields and average energies on (a) the normalized laser amplitude $a_{0}$; (b)the diameter $d$ of the waveguide; other parameters remain unchanged in the simulations. 


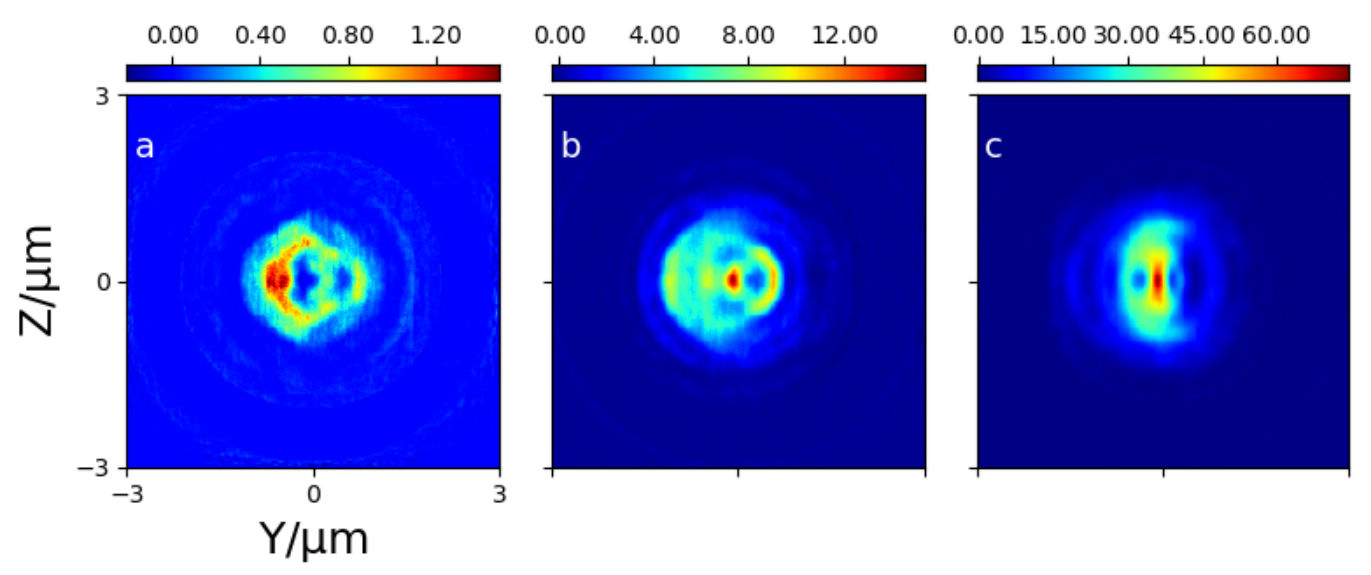

Figure 8. Subplots (a), (b), and (c) are the Poynting vector of the laser in the cross-sections of $x=9 \mu \mathrm{m}, x=13 \mu \mathrm{m}$, and $x=17 \mu \mathrm{m}$ respectively at $t=30 T$.

on the wall of the waveguide and excite the dominant hybrid mode $\mathrm{HE}_{11}$ with the high phase speed 1.02c, compared with the results in Ref .[28, 29]. This leads to oscillating forces that are several times larger and reduced oscillating periods. Therefore, photon yield with higher order of magnitude is realized.

\section{Summary}

In summary, we theoretically and numerically investigated the synchrotron radiation in a compact hollow waveguide. We demonstrated that a linearly polarized laser pulse mainly propagates inside the waveguide with the dominate hybrid mode $\mathrm{HE}_{11}$ for intense laser-wall interactions. A considerable number of electrons are extracted from the wall of the waveguide and trapped in the fields. Then, these electrons are accelerated via the longitudinal acceleration mechanism and efficiently wiggled by the transverse net forces. As a result, a bright directional X-ray beam is produced in this process. Compared with the beam from the laser-wakefield and the laser-ion channel, the X-ray beam possesses high-yield $\left(10^{11}-10^{12}\right)$ and moderate divergence $\left(5^{\circ}\right)[3,4,5,6,7,8,9,15$, $10,11,12,13,14]$. This leads to an X-ray source with considerably more photons in a single shot than achieved previously, and it has the benefits of other all optical synchrotron sources. The X-ray beam typically contains $1.2 \times 10^{-4}$ of the laser energy, compared with $10^{-6}$ from the LWFA and $10^{-5}$ from the LIC $[7,8]$. The photon flux of the synchrotron radiation is $3.5 \times 10^{21}$ photons $/ \mathrm{s} \cdot 0.1 \%$ bandwidth and the spectral brightness is $3.7 \times 10^{22}$ photons $/ \mathrm{s} \cdot \mathrm{mrad}^{2} \cdot \mathrm{mm}^{2} \cdot 0.1 \%$ bandwidth with $100 \%$ energy spread. Furthermore, the tunabilities of the radiation can be realized by adjusting the laser and waveguide parameters. Although it is possible to obtain a large spot of X-ray with moderate emittance, the high-flux source can also be used for single shot ultrafast 
High-flux X-ray photon emission by a superluminal hybrid electromagnetic mode of intense laser in a plasma

imaging assisted by the Kirkpatrick-Baez mirror[31, 32]. Meanwhile, the source may also be applied to ultrafast X-ray diffraction and absorption studies[33].

\section{Acknowledgments}

This work is supported by the National Natural Science Foundation of China(NSFC)(11605095, 11375161,11474360); Science and Technology on Plasma Physics Laboratory Foundation(6142A04020102).

\section{References}

[1] Cipiccia S, RIslam M, Ersfeld Richard Shanks P, Brunetti1 E, Vieux1 G, Yang1 X, Issac1 R C, Wiggins S M, Welsh Gregor Hand Anania M P, Maneuski D, Montgomery R, Smith G, Hoek M, Hamilton D J, Lemos N R C, Symes D, Rajeev Pattathil Pand Shea V O, Dias J M and Jaroszynski D A 2011 Nature Phys. 7(11) 867

[2] Albert F and Thomas A G R 2016 Plasma Phys. Control. Fusion 58(10) 103001

[3] Hooker S M 2003 Nature Photon. 7(10) 775

[4] Németh K, Shen B F, Li Y L, Shang H R, Crowell R, Harkay K C and Cary J R 2008 Phys. Rev. Lett. 100(9) 095002

[5] Plateau G R, Geddes C G R, Thorn D B, Chen M, Benedetti C, Esarey E, Gonsalves A J, Matlis N H, Nakamura K, Schroeder C B, Shiraishi S, Sokollik T, Van Tilborg J, Toth C, Trotsenko S, Kim T S, Battaglia M, Stöhlker T and Leemans W P 2012 Phys. Rev. Lett. 109(6) 064802

[6] Rousse A, Ta Phuoc K, Shah R, Pukhov A, Lefebvre E, Malka V, Kiselev S, Burgy F, Rousseau J P, Umstadter D and Hulin D 2004 Phys. Rev. Lett. 93(13) 135005

[7] Corde S, Ta Phuoc K, Lambert G, Fitour R, Malka V, Rousse A, Beck A and Lefebvre E 2013 Rev. Mod. Phys. 85(1) 1

[8] Kneip S, McGuffey C, Martins J L, Martins S F, Bellei C, Chvykov V, Dollar F Fonseca R, Huntington C, Kalintchenko G, Maksimchuk A, Mangles S P D, Matsuoka T, Nagel1 S R, Palmer C A J, Schreiber J, Ta Phuoc Kand Thomas A G R, Yanovsky V, Silva L O, Krushelnick K and Z N 2010 Nature Phys. 6(9) 980

[9] Schnell M, Sävert A, Landgraf B, Reuter M, Nicolai M, Jäckel O, Peth C, Thiele T, Jansen O, Pukhov A, Willi O, Kaluza M C and Spielmann C 2012 Phys. Rev. Lett. 108(7) 075001

[10] Ferri J, Davoine X, Kalmykov S Y and Lifschitz A 2016 Phys. Rev. Accel. Beams 19(10) 101301

[11] Yu T P, Pukhov A, Sheng Z, Liu F and Shvets G 2013 Phys. Rev. Lett. 110(4) 045001

[12] Kneip S, Nagel S R, Bellei C, Bourgeois N, Dangor A E, Gopal A, Heathcote R, Mangles S P D, Marquès J R, Maksimchuk A, Nilson P M, Ta Phuoc K, Reed S, Tzoufras M, Tsung F S, Willingale L, Mori W B, Rousse A, Krushelnick K and Najmudin Z 2008 Phys. Rev. Lett. 100(10) 105006

[13] Huang T W, Robinson A P L, Zhou C T, Qiao B, Liu B, Ruan S C, He X T and Norreys P A 2016 Phys. Rev. E 93(6) 063203

[14] Zhu X, Yin Y, Yu T, Shao F Q, Ge Z Y, Wang W Q and Liu J 2015 New J. Phys. 17(5) 053039

[15] Chen M, Luo J, Li F Y, Liu F, Sheng Z M and Zhang J 2016 Light Sci. Appl. 5(1) e16015

[16] Luo J, Chen M, Zeng M, Vieira J, Yu L L, Weng S M, Silva L O, Jaroszynski D A, Sheng Z M and Zhang J 2016 Sci. Rep. 629101

[17] Ji L L, Snyder J, Pukhov A, Freeman R R and Akli K U 2016 Sci. Rep. 623256

[18] Joly N Y, Nold J, Chang W, Hölzer P, Nazarkin A, Wong G K L, Biancalana F and Russell P S J 2011 Phys. Rev. Lett. 106(20) 203901

[19] Jiang S, Krygier A G, Schumacher D W, Akli K U and Freeman R R 2014 Phys. Rev. E 89(1) 013106 
High-flux X-ray photon emission by a superluminal hybrid electromagnetic mode of intense laser in a plasma

[20] Noble R J, Spencer J E and Kuhlmey B T 2011 Phys. Rev. ST Accel. Beams 14(12) 121303

[21] Yu T, Hu L, Yin Y, Shao F Q, Zhuo H, Ma Y, Yang X, Luo W and Pukhov A 2014 Appl.Phys.Lett. 105(11) 114101

[22] Martinez B, d'Humiéres E and Gremillet L 2018 Plasma Phys. Control. Fusion 60(6) 074009

[23] Lécz Z and Andreev A 2016 Phys. Plasma 33(3) 033113

[24] Russell P S J, Hölzer P, Chang W, Abdolvand A and Travers J C 2014 Nature Photon. 8(4) 278

[25] Balciunas T, Fourcade-Dutin C, Fan G, Witting T, Voronin A A, Zheltikov A, Gerome F, Paulus G, Baltuska A and Benabid F 2015 Nat. Commun. 67117

[26] Jackson J D 1999 Classical Electrodynamics(3rd Edition) (New York: John Wiley \& Sons)

[27] http://www.ccpp.ac.uk

[28] Yi L, Pukhov A, Luu-Thanh P and Shen B F 2016 Phys. Rev. Lett. 116(11) 115001

[29] Yi L, Pukhov A and Shen B F 2016 Phys. Plasmas 23(7) 073110

[30] Robinson A P L, Arefiev A V and Neely D 2013 Phys. Rev. Lett. 111(6) 065002

[31] Jarre A, Fuhse C, Ollinger C, Seeger J, Tucoulou R and Salditt T 2005 Phys. Rev. Lett. 94(7) 074801

[32] Wenz J, Schleede S, Khrennikov K, Bech M, Thibault P, Heigoldt M, Pfeiffer F and Karsch S 2015 Nat. Commun. 67568

[33] Ta Phuoc K, Fitour R, Tafzi A, Garl Tand Artemiev N, Shah R, Albert F, Boschetto D and Rousse A 2007 Phys. Plasmas 14(8) 080701 